\title{
UWB High-Contrast Robust Tomographic Imaging for Medical Applications
}

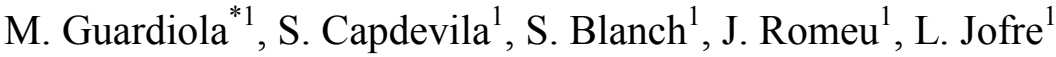

\begin{abstract}
In this paper a complete UWB Circular Tomographic System robust to high contrast or large objects, applied to Breast Tumor Detection, is presented. The main contribution of this paper is to focus on the implementation of a two degrees of freedom imaging setup in order to deal with non-symmetric objects and to demonstrate its functionality with realistic breast phantoms.
\end{abstract}

\section{INTRODUCTION}

Breast imaging using microwave frequencies has been exploited for several decades applying passive, hybrid and active approaches. Passive methods incorporate radiometers to measure the increase of temperature shown by tumors compared to normal tissue. Hybrid microwave-acoustic take advantage of microwave energy to rapidly and selectively heat tumors and detect the pressure waves generated with ultrasound transducers. Finally, active microwave imaging involves illuminating the tissue with an incident radiation and measuring the diffracted fields. This approach is able to provide satisfactorily with images of the shape and contrast in permittivities between healthy and malignant breast tissues, which is significantly greater than the few percent contrast exploited by the more conventional breast imaging modalities, such as X-ray techniques. X-ray mammography, despite being the preferred method for early-stage breast cancer detection, entails uncomfortable breast compression and exposure to low levels of ionization radiation and poses inconvenience to the recommendation of periodic breast inspections in woman over 40. Microwave signals, and in particular UWB frequency range, can overcome these drawbacks while offering a good trade-off between resolution and penetration depth.

However, active microwave approach poses an inverse scattering problem which requires a reconstruction algorithm demanding relatively high computing efforts, which was a drawback for the early imaging attempts. In spite of it, some prototypes showing good results at a single frequency were implemented, i.e. Microwave Circular Scanner [1], Microwave Planar Camera [2]. With the advent of cost effective high performance computing systems, a significant research effort in reconstruction algorithms for breast detection purposes has been made, i.e. confocal imaging techniques [3], non-linear optimization methods [4], and Fourier imaging techniques. While much progress has been made in reconstruction algorithms applied to more and more realistic breast phantom simulations, hardly ever have been tested with experimental measurements. Thus, very few efforts in implementation of measurement systems have been made, and almost always these systems use a single frequency as illumination [5]. In this paper, UWB signals are proposed as illuminating signals, since they are able to provide enhanced image resolution and clutter reduction, when compared to mono frequency approaches.

In this paper, an active UWB tomographic imaging technique based on Fourier imaging has been developed and applied to short range high-contrasted large circular objects. Section 2 outlines the basics of the tomographic formulation for circular geometries and the extension to UWB. Section 3 is devoted to describe the experimental system, and in section 4, the reconstructed images of non-symmetric breast phantoms showing the typical contrasts exhibited by real tissues, are obtained from measurements. Finally the results are discussed and some conclusions are extracted.

\section{UWB CIRCULAR TOMOGRAPHY}

The general idea behind tomographic imaging consists in placing a sufficient number of microwave sensors (transmitters and receivers) on a circular region that may completely surround the object under investigation, in order to characterize its properties by measuring the scattered fields. Each sensor is alternatively activated as a transmitter and the received signal at the rest of the sensors is captured, thus allowing to use all the information in the reconstruction procedure. At the current stage of development, bi-dimensional geometry is assumed and hence the object is supposed to be invariant in the $\mathrm{z}$-axis.

\subsection{Microwave tomography formulation}

Two configurations are commonly used for microwave tomography, planar arrays, that are based in the propagation of the spectrum of the fields, and circular arrays. When using a circular array, the incident field is a cylindrical wave, therefore in order

AntennaLab, Universitat Politècnica de Catalunya, Barcelona, Spain

e-mail: marta.guardiola@tsc.upc.edu 
to apply a conventional spectral formulation, a synthetic aperture approach is required, [6]. Using Born approximation and the reciprocity theorem, the spectrum of the contrast profile, can we obtained and expressed as:

$$
\begin{aligned}
\widetilde{C}\left(k_{0}\left(\hat{\theta}+\hat{\theta}_{0}\right)\right)= & j \omega \mu_{0} R^{2} \int_{0}^{2 \pi} \int_{0}^{2 \pi} E^{s}\left(\sigma ; \sigma_{0}\right) I(\theta-\sigma) \\
& \cdot I\left(\theta_{0}-\sigma_{0}\right) d \sigma d \sigma_{0}
\end{aligned}
$$

Where $E^{S}\left(\sigma ; \sigma_{0}\right)$ is the scattered field measured at the angular position $\sigma$, generated by an incident cylindrical wave produced by a unit source located at the angular position $\sigma_{0}, I(\alpha)$ is the current distribution of a circular array generating a plane wave towards $\alpha=0$ and $C$ is the contrast profile which is related to the complex permittivity of the object obtained through:

$$
C(\vec{r})=1-\frac{\varepsilon(\vec{r})}{\varepsilon_{0}}
$$

From (1) it can be seen that for a given frequency, we obtain a sampled version of the spectrum of the contrast profile inside of a circle of radius $2 k_{0}$, and hence the reconstructed contrast profile will consist of a smoothed version of the real contrast profile, see figure 1 .

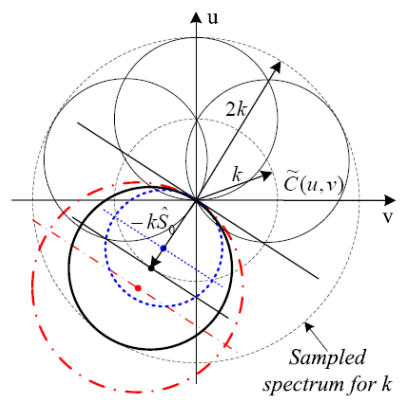

Figure 1: By measuring with a circular array for a given transmitter we will be sampling the contrast profile spectrum $\left(\widetilde{C}\left(k_{x}, k_{y}\right)\right)$ in an uncentered circle. By doing so for a set of transmitter will be sampling a circular area of radius $2 k_{0}$.

\subsection{UWB extension}

As previously said, the reconstructed contrast profile obtained using (1) consists of a low-filtered version; in order to improve the quality of the image, a set of multiple frequencies can be combined, thus allowing to obtain higher spatial frequency components of the spectrum (dotted red line of figure 1). To be able to use an UWB range of frequency and combine the information of the images obtained from each frequency, the microwave tomography algorithm needs to be extended; extra care must be taken in choosing the best frequencial combination approach, to enhance the features of interest of the individual images without having destructive interference between images. The most direct and

intuitive combination of the reconstructed images is proceed to coherently combine the different images at each frequency. This intuitive approach works well when we are doing tomography of small objects with low contrast. If the scenario consists on electrically big contrasted objects, the coherent addition of the different images may not be successful due to destructive interference. The explanation of such a behavior is found in the existence of a phase variation of the reconstructed spectrum with respect to the frequency, dependent of the electrical size of the object, and its contrast. Figure 2 presents the evolution of the phase with the frequency for a scenario with a single cylinder of different permittivities but a fixed physical size (fig. 2(a)), and fig. 2(b) for a cylinder of different diameters, but the same permittivity. The evolution of the slope clearly shows that the larger the electrical size/contrast of the body the larger the slope is, and therefore the worse a coherent reconstruction will be. It can be concluded that coherent combination can only be applied when the scenario consist of a low contrast function or a small size scatterer, which generally will not be the case for breast cancer detection. Two possibilities arise from this scenario, the first one consists in using some extra information in order to compensate such slope, or alternatively, to perform an incoherent combination of the images, that is averaging the magnitude of the images. Nevertheless, by using the magnitude averaging, although there is a loss of dielectric properties information, the size is well preserved and thus it will be applied in this paper, as it requires no extra information that could introduce some internal bias towards the reconstructed solution.

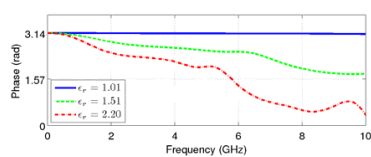

(a) Variable permittivity (20 $\mathrm{mm}$ radius)

Figure 2: Phase for the reconstructed contrast profile of a centered cylinder for different electrical sizes.

\section{EXPERIMENTAL TOMOGRAPHIC SYSTEM}

The tomographic imaging system that we are using, has been engineered to provide two degrees of freedom allowing the independent rotation of transmitting and receiving antennas and thus uncentered non-symmetric objects can be scanned. This is achieved using two high-resolution rotary stages that share the same rotating axis; the object under test and the receiving antenna are rotated concentrically and independently while the transmitting antenna remains in a fix location, see figure 3 . This configuration allows to construct two 
cylindrical virtual arrays for the transmitting and receiving antenna. Each virtual antenna array is composed by $N$ sensing points uniformly distributed along a circumference of $2 a=15 \mathrm{~cm}$ and $18 \mathrm{~cm}$ diameter, for the receiver and transmitter respectively, completely surrounding the object under test. By capturing the received signal in all sensing positions provided by the combined movement of the receiver and the object, a complete $2 \mathrm{D}$ section of a general object under test is obtained. The number of measurement points $N_{R}=N_{T}=N$ has been selected in order to avoid loss of information according to:

$$
N \geq 2 k a=2\left(\frac{2 \pi f \sqrt{\varepsilon_{r, g e l}}}{c_{0}}\right) a
$$

The measurement system has been designed taking into consideration the use of a matching gel between breast phantoms and the antennas $\left(\varepsilon_{r, g e l}\right)$, with permittivities shown in table 1. With such permittivity 128 sensors becomes the best number of sensors. Similarly the frequency domain sampling has been chosen to avoid aliasing according to:

$$
\Delta f \leq \frac{c_{0}}{4 a \sqrt{\varepsilon_{r, g e l}}}
$$

The measurements that are presented in the following section have been obtained using the system just described but using simplified phantom models of healthy and tumoral breasts, and without matching gel, the materials used for the phantoms can be observed in table 2. The size of the tumor phantom considered is about $1.6 \mathrm{~cm}$ which would be the average size of real tumors at the time of detection, [7]. The mechanization of the phantom allows to use a set of privileged positions for the breast as well as the tumor, allowing a total of six different configurations.

\begin{tabular}{r|c|c|c} 
& $\varepsilon_{\mathrm{r}}$ & $\sigma(\mathrm{S} / \mathrm{m})$ & Contrast \\
\hline Matching liquid & 9 & 0 & - \\
Skin & 6.04 & $16 \mathrm{~dB} / \mathrm{cm}$ & 0.3 \\
Breast tissue & $13-18$ & 0.83 & $0.44-1$ \\
Tumor & 51 & 1.45 & 4.6 \\
\hline
\end{tabular}

Table 1: Permittivities of breast tissue as reported in $[8,9]$ used for the measurement system.

\begin{tabular}{r|c|c|c} 
& $\varepsilon_{\mathrm{r}}$ & $\sigma(\mathrm{S} / \mathrm{m})$ & Contrast \\
\hline Matching liquid (Air) & 1 & 0 & - \\
Skin (PVC) & 2.7 & 0.0165 & 2.4 \\
Breast tissue (Air) & 1 & 0 & 1 \\
Tumor (Nylon) & 3.1 & 0.036 & 3 \\
\hline
\end{tabular}

Table 2: Material used for the preliminary breast phantom [10]. A Nylon bar has been used as it presents a contrast similar with air as that shown in a tumor with the matching liquid.



Figure 3: The experimental setup consists of two UWB antennas one of them on a rotary stage, and the other on a fixed immobile platform. The object under test is also rotating on an independent rotary stage.

\section{RESULTS AND DISCUSSION}

Three different scenarios have been analyzed in this paper and will be briefly described.

\subsection{Uncentered breast, uncentered tumor}

The first scenario consists of a hollow PVC tube representing the skin of a breast tissue placed in an uncentered position with respect to the encircling geometry. At the same time a nylon cylinder, tumor phantom, is $25 \mathrm{~mm}$ off-center from the breast phantom. The reconstruction using both UWB combination methodologies is shown in figure 4 .

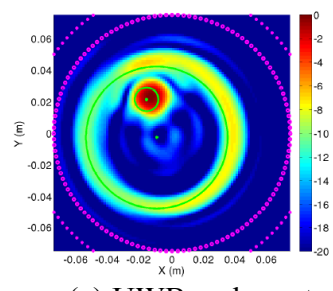

(a) UWB coherent reconstruction.

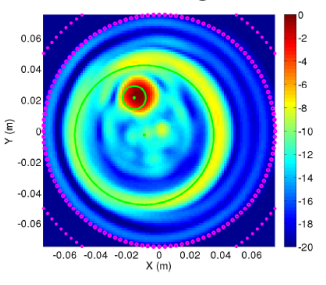

(b) UWB incoherent reconstruction
Figure 4: Reconstruction of a hollow breast phantom with a malignant tissue. The lines over the image represent the actual position of the breast and tumor.

\subsection{Uncentered healthy breast}

The second scenario consists of the same geometry than the previous measurement, but in this case the tumor phantom has been removed; figure 5 shows the results. From this and the previous reconstructions, it can be seen that both scenarios have been well reconstructed, and in both cases the characteristics that were of interest, breast and tumor location and size, have been perfectly obtained. It should be noted that in each reconstruction, both combination procedures, coherent and incoherent, have performed well in obtaining the results. The reason of this is that the electrical size of the objects is not enough as to introduce a high slope in the phase of our 
reconstructed images, and we obtain benefits from the coherent combination, removing artifacts.

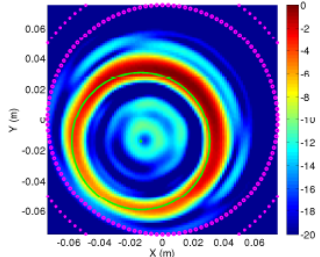

(a) UWB coherent reconstruction.

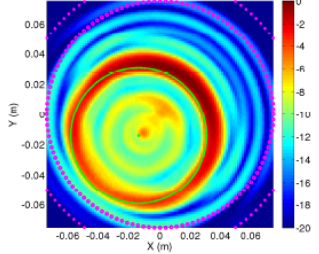

(b) UWB incoherent reconstruction
Figure 5: reconstruction of a healthy hollow breast phantom.

\subsection{Uncentered filled breast phantom. Simulation}

The data for the final scenario has been produced using a FDTD simulator. In this case the disposition is the same as the first scenario, but the PVC tube has been filled with vaseline $\left(\varepsilon_{r}=2.16\right)$, in order to introduce a dielectric media in the breast phantom, which is a more realistic approach. Figure 6 presents the reconstruction results for each of them. In this case the coherent combination is unable to obtain the tumor location, due to the destructive combination of the different images; on the other hand the incoherent reconstruction is able to return the existence of a filling media inside of the phantom, and also is capable of locating the tumor.

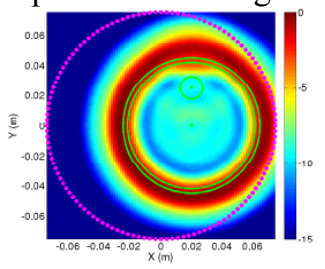

(a) UWB coherent reconstruction.

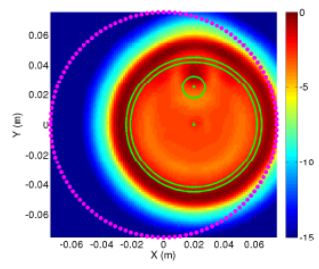

(b) UWB incoherent reconstruction
Figure 6: Reconstruction of a breast phantom with malignant tissue using simulated data.

\section{CONCLUSIONS}

A complete UWB Tomographic system applied to image of high contrast large objects has been presented, paying special attention to implementation concerns. A two degrees of freedom experimental setup, allows to synthesize two cylindrical virtual arrays for the transmitting and receiving antennas and thus adding capabilities to measure non axiallysymmetric scenarios as earlier shown. For its validation, a $2 \mathrm{D}$ breast phantom consisting of a uncentered nylon cylinder inserted into an empty PVC tube, has been conceived, obtaining accurate results for both coherent and incoherent combination procedures. By filling the PVC tube with vaseline, the phantom acts as an electrically large object, and thus only a incoherent combination approach is capable of obtaining an accurate image of the breast and tumoral tissue.

\section{Acknowledgments}

This work was supported in part by the Spanish Interministerial Commission on Science and Technology (CICYT) under projects TEC200766698-C04-01/TCM and CONSOLIDER CSD200800068 .

\section{References}

[1] L. Jofre, M. Hawley, A. Broquetas, E. de los Reyes, M. Ferrando, and A. Elias-Fuste, "Medical imaging with a microwave tomographic scanner," Biomedical Engineering, IEEE Transactions on, vol. 37, no. 3, pp. 303-312, March 1990.

[2] J. C. Bolomey, C. Pichot, and G. Gabriaud, "Planar microwave imaging camera for biomedical applications: critical and prospective analysis," Radio Science, vol. 26, pp. 541-549, 1991.

[3] X. Li and S. Hagness, "A confocal microwave imaging algorithm for breast cancer detection," Microwave and Wireless Components Letters, IEEE, vol. 11, no. 3, pp. 130-132, Mar 2001.

[4] M. Xu, A. Sabouni, P. Thulasiraman, S. Noghanian, and S. Pistorius, "Image reconstruction using microwave tomography for breast cancer detection on distributed memory machine," Parallel Processing, 2007. ICPP 2007. International Conference on, pp. 3636, Sept. 2007.

[5] P. Meaney, M. Fanning, D. Li, S. Poplack, and K. Paulsen, "A clinical prototype for active microwave imaging of the breast," Microwave Theory and Techniques, IEEE Transactions on, vol. 48, no. 11, pp. 1841-1853, Nov 2000

[6] J. Rius, C. Pichot, L. Jofre, J. Bolomey, N. Joachimowicz, A. Broquetas, and M. Ferrando, "Planar and cylindrical active microwave temperature imaging: numerical simulations," Medical Imaging, IEEE Transactions on, vol. 11, no. 4, pp. 457- 469, 1992.

[7] S. P. Poplack, A. N. Tosteson, M. R. Grove, W. A. Wells, and P. A. Carney, "Mammography in 53,803 women from the new hampshire mammography network." Radiology, vol. 217 , no. 3, pp. 832-840, Dec 2000

[8] W. T. Joines, Y. Zhang, C. Li, and R. L. Jirtle, "The measured electrical properties of normal and malignant human tissues from 50 to 900 mhz." Med Phys, vol. 21, no. 4, pp. 547-550, Apr 1994

[9] S. P. Poplack, K. D. Paulsen, A. Hartov, P. M. Meaney, B. W. Pogue, T. D. Tosteson, M. R. Grove, S. K. Soho, and W. A. Wells, "Electromagnetic breast imaging: average tissue property values in women with negative clinical findings." Radiology, vol. 231, no. 2, pp. 571580, May 2004.

[10] B. Riddle, J. Baker-Jarvis, and J. Krupka, "Complex permittivity measurements of common plastics over variable temperatures," Microwave Theory and Techniques, IEEE Transactions on, vol. 51, no. 3, pp. 727-733, Mar 2003. 